\title{
Testing the encoding elaboration hypothesis: The effects of exemplar ranking on recognition and recall
}

\author{
PAUL SCHNUR \\ Fordham University at Lincoln Center, New York, New York 10023
}

\begin{abstract}
Xwo experiments investigated the effects of exemplar ranking on retention. High-ranking exemplars are words judged to be prototypical of a given category; low-ranking exemplars art: words judged to be atypical of a given category. In Experiment 1, an incidental learning paradigm was used to measure reaction time to answer an encoding question as well as subsequent recognition. It was found that low-ranking exemplars were classified more slowly but cognized better than high-ranking exemplars. Other comparisons of the effects of categors encoding, rhyme encoding, and typescript encoding on response latency and recognition replicated the results of Craik and Tulving (1975). In Experiment 2, unanticipated free recall of five previously learned paired associate lists revealed that a list composed of low-ranking exfunplars was better recalled than a comparable list composed of high-ranking exemplars. Mrreover, this was true only when the lists were studied in the context of appropriate category cues. These findings are discussed in terms of the encoding elaboration hypothesis.
\end{abstract}

Recent experiments have demonstrated that recall and recognition are facilitated by orienting tasks which direct the learner's attention to the semantic features of words as compared to their phonemic or structural features (Craik \& Tulving, 1975; Hyde, 1973; Hyde \& Jenkins, 1969, 1973; Till \& Jenkins, 1973). For example, retention is better following an orienting task in which target words must be classified according to category membership (e.g., Is a car a type of vehicle?) than following orienting tasks in which words must be classified according to thyme (e.g., Does car rhyme with bar?) or typescript (e.g., Is car in small letters?). However, a semantic context alone is not sufficient to facilitate retention. The encoding context must also be applicable to or congruous with (Schulman, 1974) the target word. In the query, "Is a car a type of vehicle?" the target word is congruous with its context because a car is appropriately considered a vehicle. In the query, "Is a car a type of vegetable?" the target word is incongruous with its context because a car is not appropriately considered a vegetable. Typically, congruous words are better recalled and recognized than incongruous words, particularly in semantic and phonemic contexts (Craik \& Tulving, 1975; Schulman, 1974).

Craik and Tulving (1975) have proposed that "degree of encoding elaboration" provides a satisfactory

I would like to thank Fergus Craik, Adele Gottfried, Allen Gottfried, and George Potts for their helpful comments on the manuscript. A preliminary report of these findings was presented at the Eastern Psychological Association Meetings, Boston, 1977. Requests for reprints should be sent to Paul Schnur, Division of the Social Sciences, Fordham University at Lincoln Center, New York, New York 10023. explanation of these findings. According to their formulation, retention is enhanced to the degree that the orienting task (or encoding question) provides a context in which relevant descriptive attributes of the target word will be processed. Craik and Tulving (1975) argue that target words are more elaborated (and therefore better retained) in semantic as compared to nonsemantic contexts and in congruous as compared to incongruous contexts. The experiments reported below are designed to test the encoding elaboration hypothesis.

\section{EXPERIMENT 1}

According to the encoding elaboration hypothesis, retention depends upon the relation between encoding context and target word. That is, the target word is processed in terms of information provided by the context. Craik and Tulving (1975) tested retention of target words which appeared in contexts ranging from simple (e.g., "He dropped the watch") to complex (e.g., "The old man hobbled across the room and picked up the valuable watch from the mahogany table"). They found that recall of a given target word increased as a function of context complexity. In order to show that retention depends upon the relation of context to target, and not merely upon context complexity, a complementary demonstration is needed. In the present study, then, the relation between context and target is again manipulated, but context per se is held constant.

In order to manipulate the relation between encoding context and target word while maintaining the same nominal context, the present work draws upon that of 
Rosch (1975), who has provided norms for the internal structure of several categories. The internal structure of a category is given by a listing of various exemplars of that category, rank ordered in terms of how representative of the category individuals judge each exemplar to be. For example, the category "weapon" is represented, in part, by the following exemplars (and rankings): gun (1), rifle (5), knife (7), bullet (22), whip (30), fists (34), hand (52), car (57), shoes (60). Rosch (1975) demonstrated that the rankings are highly reliable and directly related to the speed with which people classify exemplar pairs as being the same or different after reading the category name.

One interpretation of this latter finding is that highranking exemplars are so similar to what is meant by the category name that the classification decision is simple, perhaps automatic. At the same time, however, it might be said that the category name does not provide much information beyond that implicit in the highranking exemplar. In other words, the target is redundant with information provided by the context and as such receives little processing. By contrast, low. ranking exemplars are less similar to what is meant by the category name (perhaps because they also exemplify other categories) and, therefore, their classification requires deliberate consideration and more time. In this case, it might be said that the category name does provide information beyond that implicit in the lowranking exemplar. The target is not fully redundant with information provided by the context, and is therefore extensively processed. This analysis (cf. Rosch \& Mervis, 1975) suggests that a low-ranking exemplar must be processed further (more elaborated) than a high-ranking exemplar to judge that it belongs to a given category. Thus, for a given category encoding context, low-ranking exemplars should be more elaborated and better retained than high-ranking exemplars.

The present experiment used an incidental learning situation to investigate the effects of category, rhyme, and typescript orienting tasks on subsequent recog. nition. The rhyme and typescript conditions were included to insure that the procedures used in this study produce the effects reported by others (e.g., Craik \& Tulving, 1975; Hyde \& Jenkins, 1969). In addition, encoding elaboration was manipulated within the category task by choosing high-, medium-, and lowranking exemplars from a given category to serve as target words. Reaction time to answer the encoding question and recognition were measured. On the basis of previous results (e.g., Craik \& Tulving, 1975), it was expected that category questions would lead to longer reaction times and better recognition than rhyme questions and these would lead to longer reaction times and better recognition than typescript questions. It was also expected on the basis of earlier work that congruous words would be better recognized than incongruous words in category and rhyme tasks, but that reaction times between congruous and incongruous words would not differ. Finally, on the basis of the above analysis of encoding elaboration, it was hypothesized that congruous low-ranking exemplars would be classified more slowly and recognized better than congruous medium-ranking exemplars, and these would be classified more slowly and recognized better than congruous high-ranking exemplars. There should be no differences in reaction times or recognition among incongruous words as a function of exemplar ranking.

\section{Method}

Twenty-four college students of both sexes volunteered to participate in the experiment. Each was tested individually in an experimental session which lasted 20 to $25 \mathrm{~min}$. They were told that the experiment concerned word perception and speed of reaction and that their task was to answer quickly and accurately questions about words by pressing a "yes" or "no" button. Half the participants pressed the "yes" button with their preferred hand and half pressed it with their nonpreferred hand (counterbalanced across all treatment conditions). An example of each type of question and its correct answer were provided, but participants were not informed of the final recognition test.

On each trial, a word was presented in a tachistoscope (Polymetric Company, Model V-0959) for 2 sec. Before presenting each word, the experimenter asked a question about it. The category question was, "Could the word be considered a type of (category name)?" The rhyme question was, "Does the word rhyme with ? The typescript question was, "Is the word in capital (small) letters?" The word was presented and reaction time recorded electronically, each trial taking approximately $12 \mathrm{sec}$. After all words had been presented in this manner, the participant was engaged in conversation for 30 to $60 \mathrm{sec}$ and then given the recognition test, which was not time limited. Finally, participants were told the purpose of the experiment, asked not to discuss it with others, and asked if they had foreknowledge of the recognition test-none had.

The stimuli were 54 common words, half appearing in uppercase letters and half appearing in lowercase letters (counterbalanced across all treatment conditions). For each participant, one-third of the words was associated with category questions, one-third with rhyme questions, and one-third with typescript questions. In addition, one-third of the words associated with category questions were high-ranking exemplars, one-third were medium-ranking exemplars, and one-third were low-ranking exemplars. High-, medium-, and low-ranking exemplars were of approximately equal frequency of occurrence in the Thorndike and Lorge (1944) norms. Within each question type (and exemplar level), half of the questions required a "yes" response (congruous), and half required a "no" response (incongruous). Words were presented in different random order to each participant.

Words were chosen from the following nine categories: fruit, furniture, vehicle, weapon, vegetable, carpenter's tool, bird, sport, clothing. Two high-ranking, two medium-ranking, and two low-ranking exemplars were selected from each category. With reference to the 7-point (1-7) rating scale used by Rosch (1975), the high-ranking exemplars had a mean rating of 1.16 , the medium-ranking exemplars had a mean rating of 3.36 , and the low-ranking exemplars had a mean rating of 5.21. Thus, a pool of 54 category words was generated, each participant receiving 18 ( 2 from each category), half requiring "yes" responses, half requiring "no" responses. Words from the above categories required a "no" response when queried by one of nine incongruous categories (weather, insect, 
entertainer, nobility, clergy, metal, flower, container, beverage). Across all participants, each of the words in the category pool, in both "yes" and "no" formats, was used equally often.

A second pool of 54 words was associated with rhyme questions and a third pool of 54 words was associated with typescript questions. Although other investigators (e.g., Craik \& Tulving, 1975) typically use the same word under each encoding format, it was not feasible to do so in the present study since words and categories were limited to those provided by Rosch (1975). As such, many exemplars were particularly difficult to thyme (e.., avocado, handkerchief). Therefore, the rhyme and typescript word pools were generated by choosing words with equal frequency of occurrence (Kučera \& Francis, 1967) to those in the category word pool. The assumption that no systematic differences in recognizability among word pools were thereby created was verified by testing six people who did not take part in the main experiment. These participants simply classified words from the three pools in response to typescript questions and then took the recognition test. Neither the effect of word pool $[1:(2,10)=1.36]$ nor response type $[F(1,5)=.08]$, nor their interaction $[F(2,10)=2.56]$ was significant.

1 tom the pool of 54 rhyme words, each participant received 18, hatf requiting a "yes" response and hall requiring a "no" response to nine thyme queries. Across all participants, each of the words in the rhyme pool, in both "yes" and "no" formats, was used equally often. From the pool of 54 typescript words, each participant received 18, half requiring a "yes" and half a "110" response. For 12 participants, the typescript question referred to capital letters; for the other 12 , it referred to small letters. Across all participants, each of the words in the typescript pool, in both "yes" and "no" formats, was used equally often.

To summarize, each participant received 54 randomly ordered words, 18 preceded by category questions ( 2 words in each of ninc categories; 6 high-, 6 medium-, and 6 low-ranking exemplars), a second group of 18 preceded by rhyme questions, and a third group of 18 preceded by typescript questions. Within each grotip, an equal number of words required "yes" and "no" responses. The recognition test consisted of the total pool of 162 words (i.e., 54 targets and 108 distractors), randomly ordered. Participants were instructed to circle only those words which had appeared in the tachistoscope. All participants received the same recognition test and none was told how many words had appeared.

\section{Results}

Classification errors during the orienting-task phase of the experiment accounted for less than $5 \%$ of all responses. Nevertheless, they were excluded and all analyses refer only to words correctly classified. In addition, the false alarm rate in the recognition test was very low (less than $3 \%$ of all responses), so that recognition performance refers only to hits.

Processing time. Table 1 (upper portion) shows mean latencies (in seconds) as a function of type of question and type of response. The only reliable effect was that of question type, with latencies increasing systematically from typescript questions to category questions. Analysis of variance of the latency scores revealed a significant effect of question type $[F(2,46)=14.59, p<.005]$, but neither the effect of response type $[F(1,23)=2.27]$ nor that of the interaction $(\mathrm{F}<1)$ was significant.

Table 2 (upper portion) shows mean latencies as a function of exemplar ranking and type of response. Among words requiring a "yes" response (congruous),
Table 1

Response Latency and Recognition Performance as a Function of Encoding and Type of Response (Experiment 1)

\begin{tabular}{cccc}
\hline \multirow{2}{*}{$\begin{array}{c}\text { Response } \\
\text { Type }\end{array}$} & \multicolumn{3}{c}{ Encoding Question } \\
\cline { 2 - 4 } & Category & Rhyme & Typescript \\
\hline Yes & \multicolumn{3}{c}{ Response Latency (sec) } \\
No & .90 & .78 & .72 \\
& .92 & .82 & .76 \\
Yes & & Proportion Recognized \\
No & .77 & .51 & .16 \\
\hline
\end{tabular}

high-ranking exemplars were classified faster than medium-ranking exemplars and these, in turn, were classified faster than low-ranking exemplars. Among incongruous words, there were no differences in classification times as a function of exemplar ranking. These conclusions are corroborated by analysis of variance which revealed a significant effect of exemplar ranking $[F(2,46)=12.04, p<.005]$, a nonsignificant effect of response type $(F<1)$, and a significant interaction $[F(2,46)=6.39, p<.005]$.

Recognition. Table 1 (lower portion) shows mean recognition scores as a function of type of question and type of response. Words associated with category questions were recognized better than words associated with rhyme questions, and these were recognized better than words associated with typescript questions. In addition, congruous words were better recognized than incongruous words, but only for category and rhyme tasks. The conclusions are substantiated by analysis of variance which revealed a significant effect of question type $[F(2,46)=87.00, p<.005]$, a significant effect of response type $[F(1,23)=46.52, p<.005]$, and a significant interaction $[F(2,46)=12.55$, $\mathrm{p}<.005]$.

Table 2 (lower portion) shows mean recognition scores as a function of exemplar ranking and response type. Among congruous words, low-ranking exemplars were best recognized, while high-ranking exemplars were worst recognized. Among incongruous words, there were no differences in recognition performance as a function of exemplar ranking. Congruous words were better recognized than incongruous words. Again,

Table 2

Response Latency and Recoginition Performance as a Function of Exemplar Ranking and Type of Response (Experiment 1)

\begin{tabular}{cccc}
\hline \multirow{2}{*}{$\begin{array}{c}\text { Response } \\
\text { Type }\end{array}$} & High & Medium & Low \\
\cline { 2 - 4 } & & Response Latency (sec) \\
Yes & .79 & .91 & 1.03 \\
No & .88 & .93 & .90 \\
& & Proportion Recognized & \\
Yes & .65 & .81 & .90 \\
No & .51 & .49 & .50 \\
\hline
\end{tabular}


analysis of variance confirmed these impressions, revealing a marginally significant effect of exemplar ranking $\quad[F(2,46)=3.17, .05<\mathrm{p}<.10]$, a highly significant effect of response type $[F(1,23)=56.54$, $\mathrm{p}<.005]$, and a marginally significant interaction $[F(2,46)=3.07, .05<p<.10]$. Individual comparisons among treatment means indicated that for congruous words, low-ranking exemplars $(p<.01)$ and mediumranking exemplars $(p<.025)$ were better recognized than high-ranking exemplars, but the former two did not differ from each other $(\mathrm{p}>.05)$.

\section{Discussion}

The results of the present experiment are consistent with the encoding elaboration hypothesis. The latency data show that category processing took longer than rhyme processing, which took longer than typescript processing, but that congruous words took no longer than incongruous words to classify. This pattern of results precisely replicates that reported by Craik and Tulving (1975). Moreover, in the context of a congruous query, high-ranking exemplars were classified faster than medium-ranking exemplars, and these were classified faster than low-ranking exemplars. There were no latency differences as a function of exemplar ranking for words presented in the context of an incongruous query. These data parallel those reported by Rosch (1975) and establish that the relation between exemplar ranking and classification time is not restricted to the priming paradigm which she used.

The recognition data are also consistent with the hypothesis. Category processing led to better recognition than rhyme processing, which in turn led to better recognition than typescript processing. In addition, superior recognition for congruous words as compared to incongruous words was observed only under category and rhyme encoding conditions. Again, the close fit to earlier data (Craik \& Tulving, 1975) is evident. Finally, for congruous encodings, low-ranking exemplars were best recognized and high-ranking exemplars worst recognized, as predicted.

This latter result, in particular, provides important confirmation of the encoding elaboration hypothesis. As outlined above, a low-ranking exemplar is presumed to be more elaborated than a high-ranking exemplar because, in the context of a given category question, the high-ranking exemplar is redundant with information provided by the context, whereas the low-ranking exemplar is relatively informative. It is noteworthy that this idea occurs repeatedly in the psychological literature of learning and memory. For example, Estes (1972) cites the conditioning studies of Egger and Miller (1962) and the concept formation studies of Trabasso and Bower (1968) to make the point, "there is considerable evidence to indicate that the activity which facilitates transition to long-term memory is initiated only if the second of two associated events resolves some uncertainty on the part of the learner" (Estes, 1972, p. 10). Despite differences in terminology, the $c$ re ideas closely resemble those implications of the encoding elaboration hypothesis delineated above.

An alternative interpretation of the present findings is that recognition is a simple function of time to answer the encoding question. Tables 1 and 2 reveal that, as response latency increases, so does recognition performance. And, in Table 2, where differences in latency do not appear for "no" responses, neither do differences in recognition. However, Table 1 also shows that there were no systematic differences in latencies between "yes" and "no" responses as a function of encoding question, but that for category and rhyme questions, recognition was better for "yes" than for "no" responses. Similarly, Table 2 shows that there were no systematic differences in latencies between "yes" and "no" responses as a function of exemplar ranking, but that recognition was superior for "yes" as compared to "no" responses. Furthermore, if recognition were due simply to processing time, then within any one condition, longer processing times should be associated with superior recognition. This possibility was examined by dichotomizing latencies for each participant in each condition into fast and slow subsets of words. Then, mean recognition proportions for the fast and slow subsets were calculated across all participants for each condition. This analysis failed to reveal any differences in recognition whatsoever between fast and slow subsets in any condition, and corroborates the results reported by Craik and Tulving (1975). Thus, it is the qualitative nature of the encoding, rather than processing time, which affects retention.

In order to test the generalizability of the effects of exemplar ranking reported above, a second experiment was undertaken, using a different paradigm.

\section{EXPERIMENT 2}

This experiment investigated the effects of exemplar ranking on retention following paired associate learning. Polson (1967) required participants to learn five successive lists of word-number pairs, where each list used the numbers 1-8 with different stimulus words. Across the five lists, however, the same response number was associated with words drawn from the same category. For example, the response " 8 " was paired with the words horse, cat, lion, bear, and dog in successive lists. Polson (1967) found that positive transfer increased progressively over the last four lists. An interpretation of these results is that participants gradually learned to use category names as mediators between exemplars and responses in successive paired associate lists. Since Polson (1967) used only highranking exemplars as stimuli, one may ask what effects variations in exemplar ranking would have in this situation. Specifically, four lists might contain high- 
ranking exemplars of each category and a fifth, lowranking exemplars. The encoding elaboration hypothesis leads to the following prediction: If after learning all lists, participants were unexpectedly asked to recall all words studied, a higher proportion of words should be given from a list of low-ranking exemplars than from a comparable list of high-ranking exemplars. The argument is that the low-ranking exemplars would be more elaborated than the high-ranking exemplars in the category context provided implicitly by the original task and, therefore, better recalled. The context is provided implicitly because the task allows participants to discover category names; in Experiment 1, category names were given by the encoding question.

\section{Method}

Twenty-one college students of both sexes volunteered to participate in the experiment. Each was tested individually in an experimental session, which lasted 30 to $40 \mathrm{~min}$. They were told that their task was to learn several lists of paired associates until each was mastered, but they were not forewarned of the final recall test.

Each paired associate list was presented using the study. test procedure. On each study triat, a word-number pair was presented in a tachistoscope (Polymetric Company, Model V-0959) for $2 \mathrm{sec}$. On each test trial, a word alone was presented in the tachistoscope for $2 \mathrm{sec}$ and the research participant was required to respond orally with the correct number (guessing if unsure). Feedback was not provided. Study trials alternated with test trials until one perfect recitation of the list was achieved. Participants were then told that a new list would appear and study trials alternated with test trials until this list was mastered. This procedure was repeated until a total of five paired associate lists had been mastered. A free recall test (no time limit) of all stimulus words was then administered. Finally, participants were asked how they had learned the lists, told the purpose of the experiment, and asked not to discuss the procedures with other students.

Participants were randomly assigned to three treatment groups. For Group $H \quad(n=7)$, the stimuli in the five lists consisted of high-ranking exemplars from nine different categories (the same as those used in Experiment 1). Across successive lists, exemplars from a given category were paired with the same response number. For example, the response "1" was paired with robin, canary, sparrow, parakeet, and dove in Lists 1-5. The category/response-number pairings and the order of presentation of the lists were randomly determined but were the same for all participants. Each study and each test trial consisted of a different random ordering of the items. The average ratings (Rosch, 1975) of the high-ranking exemplars in Lists $1-5$ were $1.23,1.19,1.38,1.31$, and 1.36. For Group L $(\mathrm{n}=7)$, Lists $1,2,3$, and 5 were the same as those used in Group H. However, the stimuli in List 4 consisted of low-ranking exemplars of the same nine categories used in the other lists. For example, the response " 1 " was paired with penguin in List 4. The average rating (Rosch, 1975) of the low-ranking exemplars was 5.36. According to the rationale outlined above, it was expected that final free recall of List 4 would be greater for Group L than for Group H. Finally, Group C $(n=7)$ was a control group to insure that any difference between Groups $H$ and $L$ could be attributed unambiguously to the relation between the implicit category context and the exemplar rankings of the target words. Therefore, Group $C$ studied the same lists as Group $L$, but the category-number pairings changed randomly from one list to the next. This should discourage the use of category encodings and eliminate the effects of exemplar ranking.
Table 3

Mean Proportion Correct in Free Recall for Lists 1-5 (Experiment 2)

\begin{tabular}{cccccc}
\hline & \multicolumn{5}{c}{ Lists } \\
\cline { 2 - 6 } Group & 1 & 2 & 3 & 4 & 5 \\
\hline H & .15 & .07 & .12 & .11 & .16 \\
L & .13 & .12 & .09 & .17 & .16 \\
C & .08 & .08 & .11 & .09 & .18 \\
\hline
\end{tabular}

\section{Results}

During original learning of Lists 1.5 , mean errors prior to criterion for Groups $\mathrm{H}, \mathrm{L}$, and $\mathrm{C}$ were 3.66, 1.63 , and 1.86 , respectively. These differences were not significant $[F(2,18)=1.51]$

Table 3 shows the mean proportion of words correctly recalled from each of the lists for Groups $\mathrm{H}$, $\mathrm{L}$, and $\mathrm{C}$. The groups did not differ in total recall, with Groups $\mathrm{H}, \mathrm{L}$, and $\mathrm{C}$ recalling an average of $12 \%, 13 \%$, and $11 \%$ of all words, respectively. Collapsing across groups, a serial position effect for lists is evident, with words from List 5 being recalled best and words from List 2 recalled worst. However, it is also clear that the effect of list position is different for the three groups. Most important for the hypothesis being tested is the superior recall of List 4 by Group $L$ as compared to that of Groups $\mathrm{H}$ and $\mathrm{C}$. Analysis of variance revealed that the effect of groups was not significant $[F(2,18)=3.03]$, but that the effect of lists was significant $[F(4,72)=10.18, p<.005]$, as was the interaction between groups and lists $[F(8,72)=3.51$, $\mathrm{p}<.005]$. Planned comparisons indicated that Group L recalled more List 4 words than Group $\mathrm{H}(\mathrm{p}<.025)$, but that Groups $\mathrm{C}$ and $\mathrm{H}$ did not differ reliably $(p>.05)$. Additional post hoc comparisons were carried out employing Duncan's multiple range test. The only differences to reach significance $(p<.05)$ were between Group $\mathrm{C}$ and Groups $\mathrm{H}$ and $\mathrm{L}$ on List 1 recall. There were no reliable differences among groups for recall of words from Lists 2, 3, or 5. Superior recall of low exemplars in Group $L$ is also shown by the fact that, in this group but not in Groups $\mathrm{H}$ and $\mathrm{C}$, recall of List 4 was higher than that of any other list. By contrast, Groups $\mathrm{H}$ and $\mathrm{C}$ both showed higher recall of List 5 than List 4 , and Group $\mathrm{H}$ also showed higher recall of List 1 than List 4.

\section{Discussion}

As predicted by the encoding elaboration hypothesis, participants who learned a paired associate list made up of low-ranking exemplars (Group L) subsequently recalled more items from this list than participants who learned a paired associate list made up of high-ranking exemplars (Group $\mathrm{H}$ ). That this effect is not attributable to inherent differences in item recallability between word lists is indicated by the performance of Group C, which studied the same words as Group L, but did not recall more List 4 words than Group $\mathrm{H}$. Thus, low- 
ranking exemplars are better recalled than high-ranking exemplars when both appear in the same encoding context. However, in the absence of a common encoding context, exemplar ranking has no effect on recall. It is clear that recall depends upon the relation between encoding context and target word.

The present experiment also demonstrates that the relation between encoding context and target word affects retention even when the context is specified implicitly. Unlike Experiment 1, where the context was specified explicitly by the category question, the paradigm used in Experiment 2 allowed participants to define their own encoding context. Not surprisingly, all participants in Groups $\mathrm{H}$ and $\mathrm{L}$ reported learning the paired associate lists by associating numbers with categories, and the recall protocols for most of these participants were organized by category. In contrast, no participants in GroupC reported learning by category-number associations, and only a few recognized the categorical relations among lists.

\section{GENERAL DISCUSSION}

The present work demonstrates that, for a given encoding context, retention is a function of the exemplar ranking of the target word. In Experiment 1, the effects of exemplar ranking were obtained on recognition following orienting tasks in which the encoding context was specified explicitly. In Experiment 2, the effects of exemplar ranking were obtained on free recall following paired associate learning in which the encoding context was specified implicitly. Thus, the effects of exemplar ranking on retention are reliable and are not limited to a single paradigm. With regard to the encoding elaboration hypothesis, the present findings complement Craik and Tulving's (1975) demonstration of the effects of context complexity on retention of a given target word.

Moreover, the present results are consistent with the idea that, in a given congruous context, low-ranking exemplars are more informative than high-ranking exemplars. Therefore, they are processed more extensively (see Table 2 ), recognized better (Table 2), and recalled better (Table 3) than high-ranking exemplars. An important question is why low-ranking exemplars are more informative than high-ranking exemplars. Borrowing from Garner's (1974) analysis of redundancy in visual pattern perception, it is suggested that, for a given context, low-ranking exemplars permit many alternative encodings, while high-ranking exemplars permit relatively few alternative encodings. Similar analyses are suggested by the work of Saltz and his colleagues (Klein \& Saltz, 1976; Saltz, 1971) and by Lockhart, Craik, and Jacoby (1975). There are several theoretical accounts which would then explain how the number of alternative encodings is related to retention (Martin, 1968; Tulving \& Thomson, 1973).
The present results might also be interpreted in terms of retrieval processes. Moscovitch and Craik (1976) proposed two retrieval hypotheses to account for the differential retention of words following category, rhyme, or typescript questions. One possibility is that the encodings themselves are differentially memorable. Thus, retention differences might be due to differential accessiblity to retrieval cues. Although this hypothesis can account for the effects of orienting task observed in Experiment 1, it would not seem applicable to the effects of exemplar ranking observed in both experiments, where the same encoding context preceded high-, medium-, and low-ranking exemplars. Moreover, if retention depended only upon accessibility of a suitable retrieval cue and if the encoding question were recalled at the time of test, then high-ranking exemplars would be more likely responses than low-ranking exemplars, since by definition, the category name is more closely associated with high- than low-ranking exemplars. The second possibility is that category encodings are more distinctive or unique than rhyme and typescript encodings. In this case, rhyme and typescript questions would be poorer retrieval cues than category questions, since the former do not specify the target encoding uniquely. Applied to the effects of exemplar ranking, it might be argued that the category name is a better retrieval cue for the uniquely encoded low-ranking exemplars than for the less uniquely encoded highranking exemplars. If the category name were recalled at the time of test, then the more unique encodings (low-ranking exemplars) would be more retrievable than the less unique encodings (high-ranking exemplars). This might be conceptualized either in terms of a von Restorff effect facilitating retrieval of unique items or in terms of a cue-overload effect (Watkins \& Watkins, 1975 ) inhibiting retrieval of less unique items.

Whether the effects of exemplar ranking operate primarily at the time of encoding or at the time of retrieval might be answered by future research. At the present time, however, an explanation of the effects of exemplar ranking does not compel this strict dichotomy. That is, the encoding specificity principle (Tulving \& Thomson, 1973) holds that a retrieval cue is effective to the extent that it reinstates the encoding context of the target. The present results are compatible with this principle and suggest further that the uniqueness of the stimulus-in-context (encoding elaboration) is a complementary factor of principal importance in retention.

\section{REFERENCES}

Craik, F. I. M., \& Tulving, E. Depth of processing and retention of words in episodic memory. Joumal of Experimental Psychology: General, 1975, 104, 268-294.

Egger. M. D.. \& Miller, N. E. Second ary reinforcement in rats as a function of information value and reliability of the stimulus. Joumal of Experimental Psychology, 1962. 64. $97 \cdot 104$. 
Esres. W. K. Learning theory and mental dewhynent. New York: Acialemic Press. 1972.

Garner. W. R. The processing of infirmation and stucture. Potomac. Md: Lawrence Frlbaum. 1974.

HYDE. T. S. Difterential effects of effort and type of orienting task on recall and organization of highly associated words. fonmal of Fiperimental Psichology. 1973, 77, $111-113$.

HrDe. T. S. \& Jenkins, J. J. Differential effects of incidental tasks on the organization of recall of a list of highl. associated words. Journal of Experimental Psichologi. 1469. 82. 472.481.

Hyde, T. $\$$. d Jenkins. J. J. Fecall for words as a function of scmantic, graphic and syntactic orienting tasks. Journal of V'rbal Le'aming and Verbal Behavior. 1973, 12. $f-1 \cdot+30)$

Kikix. K., at Saltz. E. Specifying the mechanisms in a lesels-ofpresessing approach to memory. Journal of Fxperimental Psichology: Human Leaming and Memory. $10^{-6} 6.6,0^{-1} 1-6^{-19}$

Kuirat. H. d Francis. W. N. Computational analysis of merm-dur American English. Providence, R.I: Brown lnisersity Press. 1967.

I. o hH+RI. R. S., Craik, F. I. M., \& Jacoby, L. L. Depth of processing in recognition and recall: Some aspects of a general menory system. In J. Brown (Ed.). Recognition cimal recull. London: Wiley. 1975.

Martis. E. Stimulus meaningfulness and paired-associate transfer: An encoding variability hypothesis. Psychological Kevien', 1968, 75, 421-441.

Moscovitch. M.. \& Craik. F. I. M. Depth of processing. retrieval cues and uniqueness of encoding as factors in recall. Journal of Verbal Learning and Verbal Behavior. $19 \%, 15,447.458$.
Polson. P. G. A quantitative study of the concept identification and paired-associates learning processes in the Hull paradigm. Unpublished doctoral thesis. Indiana University. 1967.

Rosch, E. Cognitive representations of semantic categories. Journal of Experimental Psychology: General. 1975, 104. 192.233 .

Rosch, E., \& Mervis, C. B. Family resemblances: Studies in the internal structure of categories. Cognitive Psychology. 1975, 7, 573-605.

SAl.TZ. E. The cognitive bases of human learning. Honewood. Ill: Dorsey. 1971.

Schliman. A. 1. Memory for words recently classified. Memony \& Cognition. 1974, 2, 47-52.

ThORNDIKE. E. L., \& LoRGE. I. The teacher's nord book of 30.000 words. New York: Bureau of Publications. Columbia University, 1944.

Til., R. E., \& Jenkins, J. J, The effects of cued orienting tasks on the tree recall of words. Joumal of Verbal Learning and Verbal Behavior, 1973, 12, 489-498.

Trabasso. T., \& Bower, G. H. Attention in learning. New York: Wiley, 1968.

Tutring, E., \& Thomson, D. M. Encoding speciticity and retrieval processes in episodic memory. Psychological Revicu, 1973, 80, 352-373.

Watkins, O. C.. \& Watkins, M. J. Buildup of proactive inhibition as a cue-overload effect. Journal of Experimental Psychology: Human Learning and Memony. 1975. 104. 442.452 .

(Received for publication February 25, 1977; accepted July 5, 1977.) 